Check for updates

Cite this: Mater. Adv., 2022, 3,554

Received 14th October 2021 Accepted 6th November 2021

DOI: $10.1039 / \mathrm{d} 1 \mathrm{ma} 00954 \mathrm{k}$

rsc.li/materials-advances

\section{Simple non-fused small-molecule acceptors with bithiazole core: synthesis, crystallinity and photovoltaic properties $\dagger$}

\author{
Jiali Guo, ${ }^{a}$ Wei Tang, ${ }^{\text {ab }}$ Yingshuang Zhang, ${ }^{a}$ Can Qian, ${ }^{a}$ Junfeng Wang, ${ }^{a}$ \\ Hua Tan (D) *a and Weiguo Zhu (D)*a
}

\begin{abstract}
It is well-known that most high-efficiency small-molecule acceptors (SMAs) contain a big fused-ring central core. However, their syntheses are multistep and complex. The development of high-efficiency simple non-fused SMA is of great significance for organic solar cells (OSCs). In this study, 4,4' -dimethyl2,2'-bithiazole was primarily used as a weak electron-accepting $\left(A_{w}\right)$ core to construct a type of nonfused SMAs of BTz-4F-1 and BTz-4Cl-1 with an $A_{w}(\pi-A)_{2}$ architecture, in which halogenated (fluorinated or chlorinated) cyanoindanone was used as a strong electron-accepting (A) terminal. For comparison, an analogue BTz-4F-0 with 2,2'-bithiazole was synthesized. The effect of methylation in bithiazole and halogenation in cyanoindanone on solubility, crystallinity, morphology and photovoltaic properties was primarily studied. It was found that both SMAs with dimethylbithiazole exhibited a significantly increasing solubility than BTz-4F-0. The fluorinated BTz-4F-1 exhibited an adjustable crystallinity and an increasing solu-bility in comparison with the chlorinated BTz-4Cl-1. Furthermore, BTz-4F-1 exhibited better morphological and photovoltaic properties than $\mathrm{BTz}-4 \mathrm{Cl}-1$ in the bulk heterojuncation OSCs using polymer $\mathrm{J71}$ as the donor. The obtained power conversion efficiency in the BTz-4F-1 based OSCs is 1.46 times higher than that of the BTz-4Cl-1 based ones. However, the BTz-4F-0 based OSCs were not obtained by a solution process owing to the lower solubility of BTz-4F-0. This study indicates that the crystallinity, morphology and photovoltaic properties of SMAs can be managed by central methylation and terminal fluorination strategies.
\end{abstract}

\section{Introduction}

Organic solar cells (OSCs) have become one of the most promising solar energy utilization technologies owing to their many advantages, such as low cost, lightweight, and flexible devices. $^{1-3}$ Their power conversion efficiencies (PCEs) have been continuously updated due to the invention of nonfullerene small-molecule acceptors (SMAs) in the past several years. $^{4-10}$ In comparison with fullerene acceptors, SMAs have more potential in tuning energy levels, absorption spectra, and molecular arrangement, which were rapidly developed by designing and modifying molecular structures, such as the

\footnotetext{
${ }^{a}$ School of Materials Science and Engineering, Jiangsu Engineering Laboratory of Light- Electricity-Heat Energy-Converting Materials and Applications, Jiangsu Collaborative Innovation Center of Photovoltaic Science and Engineering, National Experimental Demonstration Center for Materials Science and Engineering, Changzhou University, Changzhou, 213164, P. R. China. E-mail: zhuwg18@126.com, tanhua815@126.com

${ }^{b}$ College of Chemistry, Xiangtan University, Xiangtan, 411105, P. R. China $\dagger$ Electronic supplementary information (ESI) available. See DOI: 10.1039/ d1ma00954k
}

central core, end-capping groups, and branched side chains. ${ }^{11-14} \mathrm{Up}$ to now, most of the reported SMAs have an A-D-A or A-A' $\mathrm{DA}^{\prime}-\mathrm{A}$ skeleton, which contains a big fused-ring electron-donating (D) core (or a rigid $\mathrm{A}^{\prime} \mathrm{DA}^{\prime}$ core) and two strong electron-accepting (A) terminal groups. The fused-ring core is available to form a big plane molecular skeleton to promote carrier transport and enhance absorption. ${ }^{15-19}$ Thus, the PCE of single-layer OSCs based on the above-mentioned SMAs has exceeded $18 \% .^{9,10}$ Nevertheless, the fused-ring core requires multi-step synthesis, which leads to a high cost in practical applications. Therefore, it is crucial to develop simple-structure and high-efficiency SMAs in the future.

In order to achieve this goal, researchers have invented a strategy to construct simple- structure SMAs with an A-D-A framework, in which simple fused-ring or non-fused-ring aromatic units were employed as the electron-donating (D) units. $^{20-28}$ For instance, Chen et al. took fluorobenzene and alkoxybenzene as the $\mathrm{D}$ units to construct simple-structure non-fused-ring SMAs and obtained PCE over 10\% in bulkheterojunction OSCs. ${ }^{29}$ Our group also developed another strategy to construct simple-structure SMAs with the $A_{w}\left(\pi-A^{\prime}-A^{\prime \prime}\right)_{2}$ 
framework. The simple thiazolothiazole (TTz) and bithiazole (BTz) were primarily used as a weak electron-accepting $\left(\mathrm{A}_{\mathrm{w}}\right)$ central unit, and two adjacent electron-accepting units $\left(\mathrm{A}^{\prime}-\mathrm{A}^{\prime \prime}\right)$ were employed as the terminal units, respectively. ${ }^{22,27,30}$ As expected, the improved PCE was obtained in the OSCs using $\mathrm{A}_{\mathrm{w}}\left(\pi-\mathrm{A}^{\prime}-\mathrm{A}^{\prime \prime}\right)_{2}$-type TTz1 as the acceptor and polymer J71 as the donor. However, most of these high-efficiency simple SMAs used cyclopentadithiophene as spacers or benzothiadiazole as one of two adjacent terminal electron-accepting units, which also involve a multi-step synthesis and complex purification. Therefore, it is necessary to develop novel simpler non-fused SMAs and study their structure-property relationship for high-efficiency OSCs.

In 2020, Bo et al. used tetrathiophene as the central electrondonating unit to construct non-fused-ring SMAs and obtained PCE over $10 \%$ in bulk-heterojunction OSCs. ${ }^{31}$ In 2021, Chen et al. used thiophenes with two-dimensional phenyl side chains and alkoxybenzene as the central unit to construct fully non-fused-ring SMAs and obtained single-junction OSCs with a PCE up to $12.76 \%$, which is the highest PCE reported to date for non-fused-ring SMAs. ${ }^{32}$ However, most non-fused SMAs have not exhibited a comparable PCE in comparison with the big fused-ring SMAs. It remains a great challenge to prepare new non-fused-ring SMAs for high-efficiency OSCs.

There are many factors that influence the photovoltaic parameters of SMAs in OSCs. Their chemical structures and morphologies in the active layer are suggested to be crucial factors. Hence, it is imperative to systematically develop new non-fused-ring SMAs and study the influence of both factors on the photovoltaic properties of OSCs. With this in mind, in this study, we designed and synthesized a type of non-fused-ring SMAs with an $\mathrm{A}_{\mathrm{w}}(\pi-\mathrm{A})_{2}$ architecture, in which a type of nonfused-ring aromatic units of 2,2'-bithiazole and 4,4'-dimethyl$2,2^{\prime}$-bithiazole was designed as the $\mathrm{A}_{\mathrm{w}}$ central unit, and halogenated lcyanoindanone was used as the terminal electron-accepting (A) units. The effect of methylation in central bithiazole and halogenation (fluorination or chlorination) in terminal cyanoindanone were studied, primarily in terms of crystallinity, solubility, morphology and photovoltaic properties. The designed molecular structures of non-fused-ring SMAs for BTz-4F-0, BTz-4F-1 and BTz-4Cl-1 are shown in Fig. 1 . To the best of our knowledge, few non-fused-ring SMAs with an $A_{w}(\pi-A)_{2}$ architecture have been reported using a non-fused-ring electronaccepting unit as the central unit. This study indicates that methylation in central bithiazole and halogenation in terminal cyanoindanone plays an important role in crystallinity, solubility, morphology and photovoltaic properties for their SMAs in OSCs.

\section{Results and discussion}

\subsection{Synthesis and thermal properties}

The synthetic routes of $\mathrm{BTz}-4 \mathrm{~F}-0, \mathrm{BTz}-4 \mathrm{~F}-1$ and $\mathrm{BTz}-4 \mathrm{Cl}-1$ are shown in Scheme 1. The experimental details and structural characterizations are described in the ESI. $\dagger$ It is found that three $A_{\mathrm{w}}(\pi-\mathrm{A})_{2}$-type SMAs can be easily synthesized in three steps at a low cost. Both methylated SMAs of BTz-4F-1and BTz4Cl-1 have better solubility than the non-methylated SMA of $\mathrm{BTz}-4 \mathrm{~F}-0$, meaning methylation in central bithiazole is available to increase solubility for SMAs. However, two methylated SMAs exhibited different dissolving capacities in chloroform and chlorobenzene. BTz-4F-1 easily dissolved at room temperature, while BTz-4Cl-1 dissolved under heating conditions in the above two solvents. The reduced solubility of BTz-4Cl-1 is mainly contributed to the increased molecular self-aggregation, resulting from a larger atomic radius of the chlorine atom. Thermogravimetric analysis (TGA) results further showed that the decomposition temperatures $\left(T_{\mathrm{d}}\right)$ of BTz-4F-0, BTz- $4 \mathrm{~F}-1$ and BTz-4Cl- 1 at $5 \%$ weight loss were 344,345 and $366{ }^{\circ} \mathrm{C}$, respectively. Therefore, chlorination in terminal cyanoindanone was available to enhance the thermal stability of the SMAs (Fig. S1, ESI $\dagger$ ).

\subsection{Photophysical and electrochemical properties}

The normalized UV-Visible absorption spectra of BTz-4F-0, BTz$4 \mathrm{~F}-1$ and $\mathrm{BTz}-4 \mathrm{Cl}-1$ in $\mathrm{CHCl}_{3}$ solution and thin film are shown in Fig. 2, and the corresponding absorption data are summarized


Fig. 1 Molecular structures of polymer donor J71, small-molecule acceptors BTz-4F-0, BTz-4F-1 and BTz-4Cl-1. 


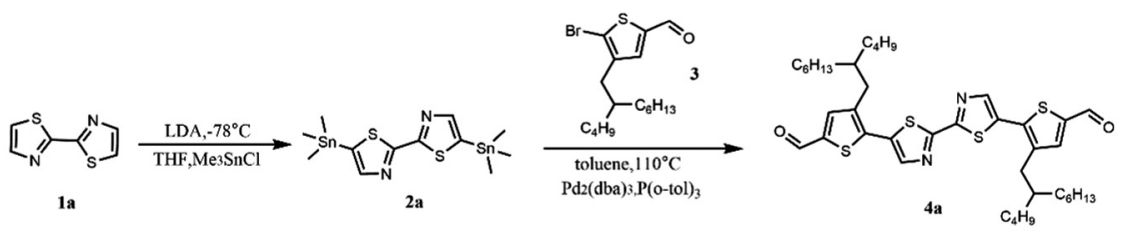<smiles>CC(C)Cc1cc(C=O)sc1-c1cnc(-c2ncc(-c3cc(C=O)sc3CC(C)C)s2)s1</smiles>

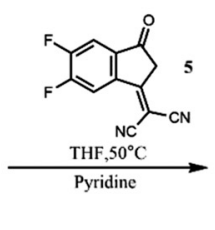

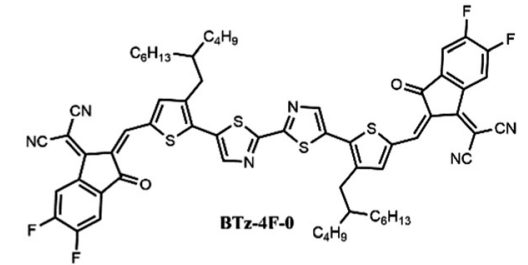<smiles>Cc1csc(-c2nc(C)cs2)n1</smiles>

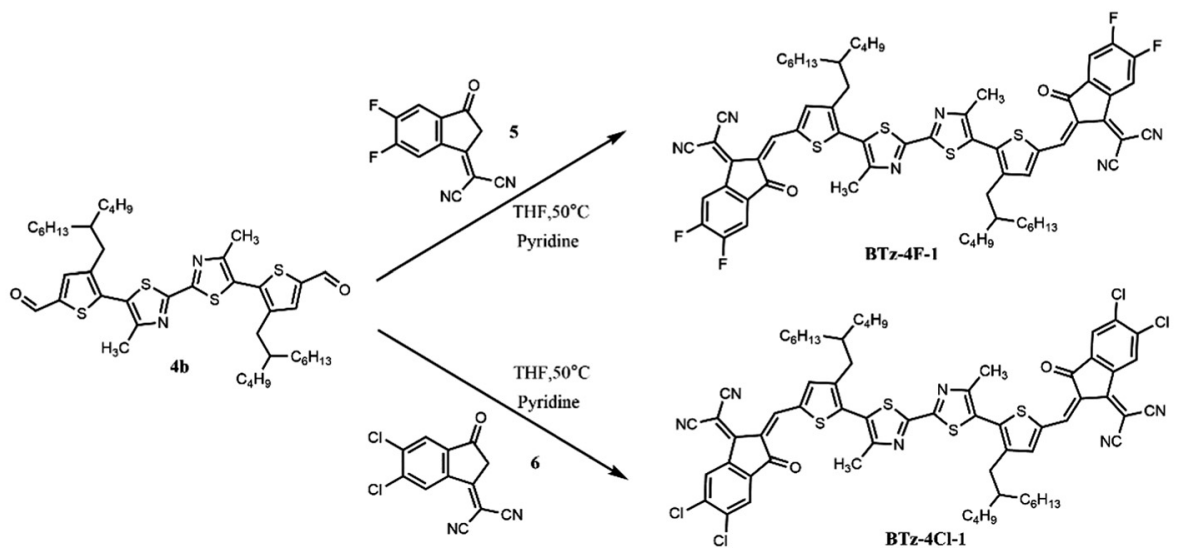

Scheme 1 Synthetic route of BTz-4F-0, BTz-4F-1 and BTz-4Cl-1.

(a)

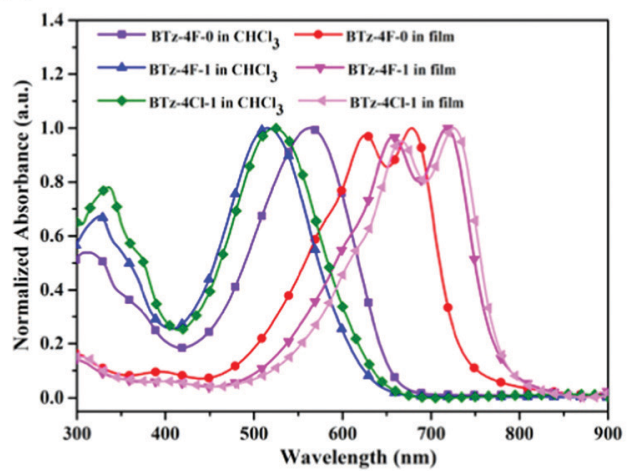

(b)

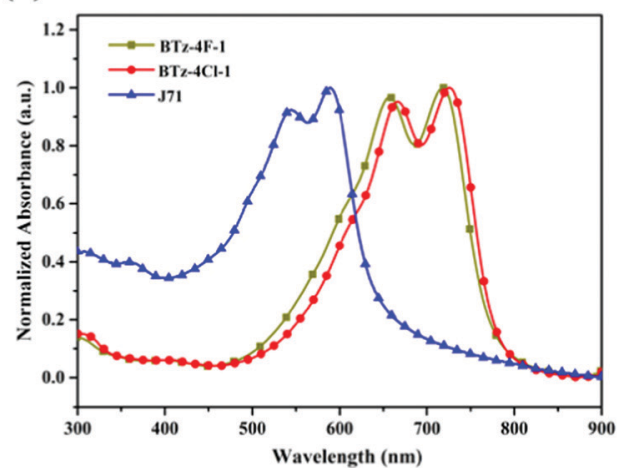

Fig. 2 (a) UV-Vis absorption spectra of BTz-4F-0, BTz-4F-1 and BDT-4Cl-1 in chloroform and thin films, (b) the absorption spectra of BTz-4F-1, BDT-4Cl-1 and $\mathrm{J71}$ in thin film.

in Table 1. It is observed that BTz-4F-1 and BTz-4Cl-1 exhibited similar absorption curves with two strong absorption bands from $300 \mathrm{~nm}$ to $650 \mathrm{~nm}$ in a chloroform solution $\left(10^{-5} \mathrm{M}\right)$ at room temperature. From the solvent to the film, BTz-4F-1 and
BTz-4Cl-1 exhibited a significant red-shifted absorption profile by about $200 \mathrm{~nm}$ owing to strong intermolecular $\pi-\pi$ interactions in the solid state. ${ }^{33}$ The non-methylated BTz-4F-0 exhibits a significantly red-shifted absorption profile by $48 \mathrm{~nm}$ in 
Table 1 Absorption and electrochemical properties of BTz-4F-0, BTz-4F-1 and BTz1-4Cl-1

\begin{tabular}{|c|c|c|c|c|c|c|c|c|}
\hline \multirow[b]{2}{*}{ Acceptors } & \multicolumn{2}{|l|}{$\lambda_{\max }(\mathrm{nm})$} & \multirow[b]{2}{*}{$\varepsilon_{\max }\left(\times 10^{5} \mathrm{M}^{-1} \mathrm{~cm}^{-1}\right)$} & \multirow[b]{2}{*}{$\lambda_{\text {onset }}(\mathrm{nm})$} & \multirow[b]{2}{*}{$E_{\mathrm{g}}^{\mathrm{opt} c}(\mathrm{eV})$} & \multirow[b]{2}{*}{$E_{\text {ox }} / E_{\text {red }}(\mathrm{V})$} & \multirow[b]{2}{*}{$E_{\text {HOMO }} / E_{\text {LUMO }}(\mathrm{eV})$} & \multirow[b]{2}{*}{$E_{\mathrm{g}}^{\mathrm{ec} d}(\mathrm{eV})$} \\
\hline & Solution $^{a}$ & Film $^{b}$ & & & & & & \\
\hline BTz-4F-0 & 312,563 & 626,678 & 1.10 & 734 & 1.69 & $1.80 /-0.15$ & $-6.03 /-4.08$ & 1.95 \\
\hline BTz-4F-1 & 326,515 & 657,718 & 1.52 & 773 & 1.60 & $1.65 /-0.30$ & $-5.88 /-3.93$ & 1.95 \\
\hline BTz-4Cl-1 & 336,523 & 667,726 & 1.01 & 784 & 1.58 & $1.76 /-0.20$ & $-5.99 /-4.03$ & 1.96 \\
\hline
\end{tabular}

comparison with the methylated BTz-4F-1 in $\mathrm{CHCl}_{3}$ solution. However, BTz-4F-0 exhibits a blue-shifted absorption profile by about $40 \mathrm{~nm}$ in comparison with the methylated BTz-4F-1 in a thin film. It means that methylation in the central bithiazole can make its SMAs exhibit stronger intermolecular interaction in a neat film owing to the electron-donating effect of the methyl group. Moreover, BTz-4Cl-1 thin film exhibited a red-shifted absorption onset by $11 \mathrm{~nm}$ compared to the BTz-4F-1 thin film owing to the higher dipole effect of the chlorine atom. ${ }^{34}$

Fig. 3a displays cyclic voltammograms of BTz-4F-0, BTz-4F-1 and BTz-4Cl-1 in their neat films measured by cyclic voltammetry (CV), and their resulting CV data are summarized in Table 1. It is found that the onset oxidation and reduction potentials $\left(E_{\mathrm{ox}} / E_{\mathrm{red}}\right)$ were $1.80 /-0.15(\mathrm{~V} / \mathrm{V})$ for BTz-4F-0, 1.65/ $-0.30(\mathrm{~V} / \mathrm{V})$ for BTz-4F-1 and $1.76 /-0.20(\mathrm{~V} / \mathrm{V})$ for BTz-4Cl-1. According to the empirical formula, HOMO and LUMO energy levels $\left(E_{\mathrm{HOMO}} / E_{\mathrm{LUMO}}\right)$ were calculated to be $-6.03 /-4.08(\mathrm{eV} / \mathrm{eV})$ for BTz-4F-0, $-5.88 /-3.93(\mathrm{eV} / \mathrm{eV})$ for BTz$4 \mathrm{~F}-1$ and $-5.99 /-4.03(\mathrm{eV} / \mathrm{eV})$ for $\mathrm{BTz}-4 \mathrm{Cl}-1$, respectively. It is apparent that the methylated BTz-4F-1 exhibited slightly increasing HOMO and LUMO energy levels in comparison with the nonmethylated BTz-4F-0. In contrast, chlorination instead of fluorination in terminal cyanoindanone can lower the HOMO and LUMO energy levels for the methylated SMAs. Therefore, the synergistic effect of the terminal halogenation and central methylation can manage the energy level of SMAs to match well with polymer J71 donor material. Thus, it can make the BTz-4F-1 based OSCs obtain a higher open circuit voltage $\left(V_{\text {oc }}\right)$, further exhibiting a higher PCE. ${ }^{35-37}$

\subsection{Theoretical calculation}

Fig. 4 shows the molecular geometries and frontier molecular orbitals of BTz-4F-0, BTz-4F-1 and BTz-4Cl-1 obtained from density functional theory (DFT) calculations. It is observed that both methylated BTz- $4 \mathrm{~F}-1$ and BTz-4Cl-1, as per BTz- $4 \mathrm{~F}-0$, have good planarity from the side view and top view of their optimal molecular structures. The torsion angle is almost zero between two thiazole units, the thiazole unit and the adjacent thiophene $\pi$ bridge, and between the thiophene $\pi$ bridge and the terminal group. It indicates that methylation in the central bithiazole has little influence on the molecular plane architecture. Both methylated SMAs also have good planarity and a strong inter-molecular $\pi-\pi$ stacking effect, which is available to facilitate charge transfer. ${ }^{38-41}$ However, a smaller twist of angle was observed in BTz-4Cl-1 rather than BTz-4F-1 by theoretical calculation. It is suggested that high atomic mass halogens are available to improve the planarity and charge transport properties of conjugated materials through a heavy atom effect. ${ }^{42}$ Therefore, the BTz-4Cl-1 with chlorine atom exhibited better planarity than BTz-4F-1 with the fluorine atom, which is consistent with a smaller torsion angle of BTz-4Cl-1. Here, a smaller twist angle makes BTz-4Cl-1 exhibit a decreasing solubility than BTz-4F-1. From the electron cloud density distribution diagram of the frontier molecular orbitals, it is found that three molecules exhibited similar electron cloud density distributions. They are delocalized in the entire molecular backbone for the LUMOs and mainly distributed on the bithiazole core and thiophene $\pi$ bridge for the HOMOs. It indicates that three SMAs have a particular ICT effect. The calculated HOMO and LUMO levels are respectively -5.90 and $-3.73 \mathrm{eV}$ for BTz-4f- $0,-5.77$ and $-3.65 \mathrm{eV}$ for BTz$4 \mathrm{~F}-1$, and -5.82 and $-3.73 \mathrm{eV}$ for BTz- $4 \mathrm{Cl}-1$, which are in good agreement with those corresponding values measured by the $\mathrm{CV}$ method. (a)

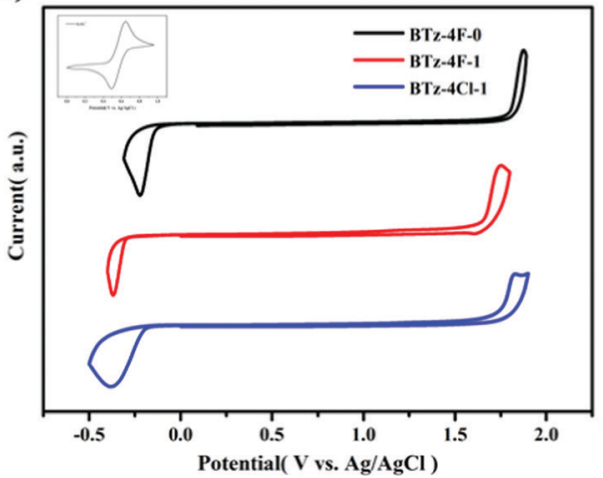

(b)

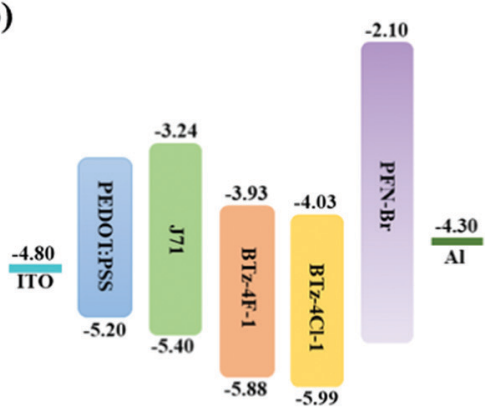

Fig. 3 (a) Cyclic voltammogram of the BTz-4F-0, BTz-4F-1 and BTz-4Cl-1 films and (b) their energy diagram relative to the vacuum level. 
BTz-4F-0

Side

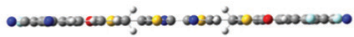

Top

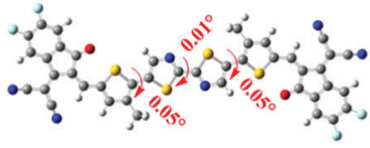

LUMO
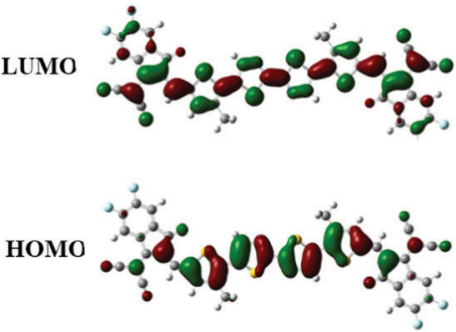

BTz-4F-1


BTz-4Cl-1
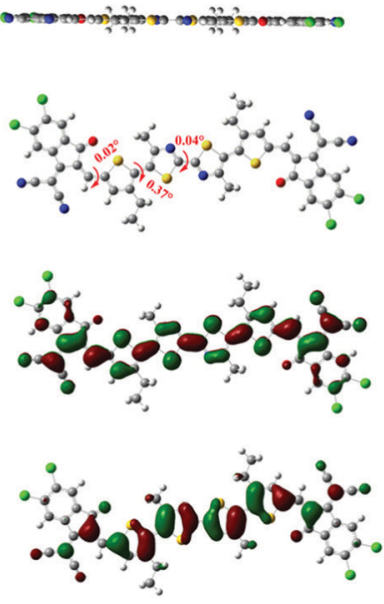

Fig. 4 Molecular geometries and frontier molecular orbitals of BTz-4F-0, BTz-4F-1 and BTz-4Cl-1.

\subsection{Photovoltaic properties}

The OSCs were made by the solution process using $\mathrm{BTz}-4 \mathrm{~F}-1$ and $\mathrm{BTz}-4 \mathrm{Cl}-1$ as the acceptor and polymer $\mathrm{J71}$ as the donor to deeply study the influence of halogenation in terminals on the photovoltaic performance. The cell structure is ITO/PEDOT: PSS/active layer/PFN-Br/Al. Similar OSCs have not been obtained by the solution process using BTz-4F-0 as the acceptor owing to the lower solubility of BTz-4F-0. Fig. 5 shows the $J-V$ curves and external quantum efficiencies (EQE) spectra of the optimized J71: BTz-4F-1 and J71: BTz-4Cl-1 based solar cells. ${ }^{43}$ The resulting optimal photovoltaic data are listed in Table 2. It is found that the BTz-4Cl-1-based device showed a PCE of $3.93 \%$ with a $V_{\mathrm{oc}}$ of $0.80 \mathrm{~V}$, a short-circuit current $\left(J_{\mathrm{sc}}\right)$ of $9.66 \mathrm{~mA} \mathrm{~cm}^{-2}$ and a fill factor (FF) of $51.0 \%$. In contrast, the optimal BTz-4F-1-based OSCs showed better photovoltaic properties than the optimal BTz-4Cl-1-based OSCs. The PCE of $5.72 \%$ with a $V_{\mathrm{oc}}$ of $0.84 \mathrm{~V}, J_{\mathrm{sc}}$ of $12.28 \mathrm{~mA} \mathrm{~cm} \mathrm{~cm}^{-2}$ and a $\mathrm{FF}$ of $55.3 \%$ was obtained in optimal BTz-4F-1-based OSCs, which was 1.46 times higher than that of BTz-4Cl-1 based ones. Higher EQE of $56 \%$ observed for the optimal J71: BTz-4F-1 blend further supported the result of higher PCE for the optimal OSCs based on the J71: BTz-4F-1 blend, in which the J71: BTz-4Cl-1 blend exhibited a lower EQE of $42 \%$. The higher EQE meant that the J71: BTz-4F-1 blend has a higher photoresponse efficiency, which improves $J_{\mathrm{sc}}$ for the J71: BTz-4F-1based OSCs.

\subsection{Charge mobility and film morphology}

The hole-only and electron-only devices were further fabricated to study the carrier-transporting properties of the photosensitive layer using the space-charge-limited current (SCLC) method. ${ }^{44}$ Fig. 6 shows the $J^{1 / 2}-V$ plots of these hole-only and electron-only devices based on the J71: BTz-4F-1 and J71: BTz4Cl-1 blends. For the J71: BTz-4Cl-1 devices, the calculated electron mobility and hole mobility are $3.50 \times 10^{-5} \mathrm{~cm}^{2} \mathrm{~V}^{-1} \mathrm{~s}^{-1}$ and $8.01 \times 10^{-5} \mathrm{~cm}^{2} \mathrm{~V}^{-1} \mathrm{~s}^{-1}$. In contrast, increasing electron mobility of $5.16 \times 10^{-5} \mathrm{~cm}^{2} \mathrm{~V}^{-1} \mathrm{~s}^{-1}$ and hole mobility of $8.95 \times$ $10^{-5} \mathrm{~cm}^{2} \mathrm{~V}^{-1} \mathrm{~s}^{-1}$ are observed for the devices based on the J71: BTz-4F-1 blends, which were slightly higher than those corresponding values for the devices based on the J71: BTz-4Cl-1 blends. It further supports the truth why the devices based on the J71: BTz-4F-1 blends exhibited higher FF. (a)

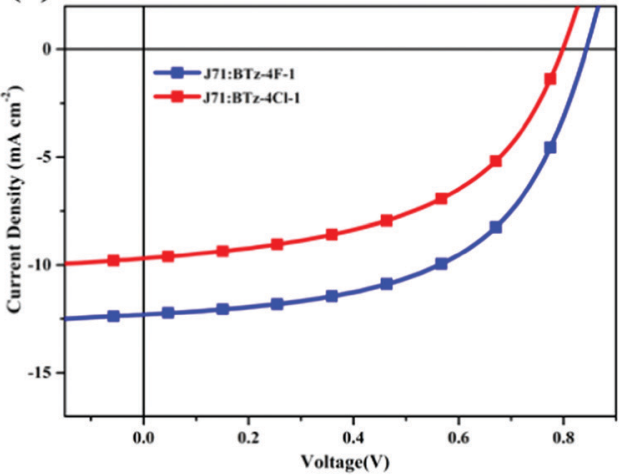

(b)

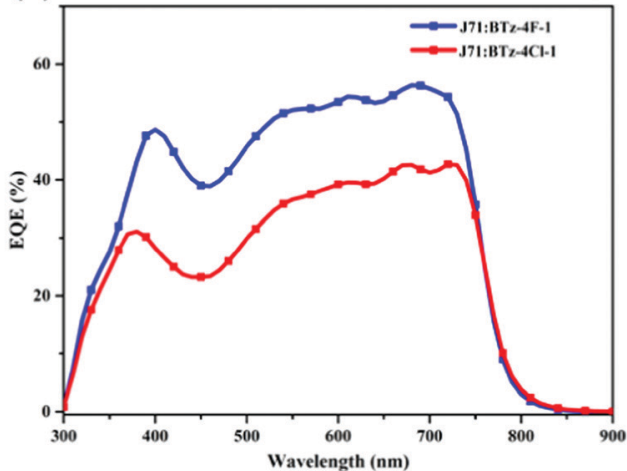

Fig. 5 (a) J-V curves and (b) EQE spectra for the BTz-4F-1 and BTz-4Cl-1 based OSCs using polymer J71 as the donor at optimized process conditions. 
Table 2 The photovoltaic properties of the BTz-4F-1 and BTz-4Cl-1 based OSCs using polymer $\mathrm{J71}$ as the donor at optimized process conditions

\begin{tabular}{|c|c|c|c|c|c|c|c|}
\hline Active layer & DIO ratio (\%) & $V_{\mathrm{oc}}(\mathrm{V}) J_{\mathrm{sc}}\left(\mathrm{mA} \mathrm{cm}^{-2}\right)$ & Cal. $J_{\mathrm{sc}}\left(\mathrm{mA} \mathrm{cm} \mathrm{cm}^{-2}\right)$ & $\mathrm{FF}(\%)$ & $\operatorname{PCE}^{a}(\%)$ & $\mu_{\mathrm{h}} \times 10^{5}\left(\mathrm{~cm}^{2} \mathrm{~V}^{-1} \mathrm{~s}^{-1}\right)$ & $\mu_{\mathrm{e}} \times 10^{5}\left(\mathrm{~cm}^{2} \mathrm{~V}^{-1} \mathrm{~s}^{-1}\right)$ \\
\hline J71: BTz-4F-1 & 1.0 & 12.28 & 12.28 & 55.3 & $5.72(5.66 \pm 0.08)$ & 8.95 & 5.16 \\
\hline J71: BTz-4Cl-1 & 1.0 & 0.80 & 9.66 & 51.0 & $3.93(3.61 \pm 0.13)$ & 8.01 & 3.50 \\
\hline
\end{tabular}

${ }^{a}$ Value in parentheses donotes the averaged PCE obtained from 10 devices.
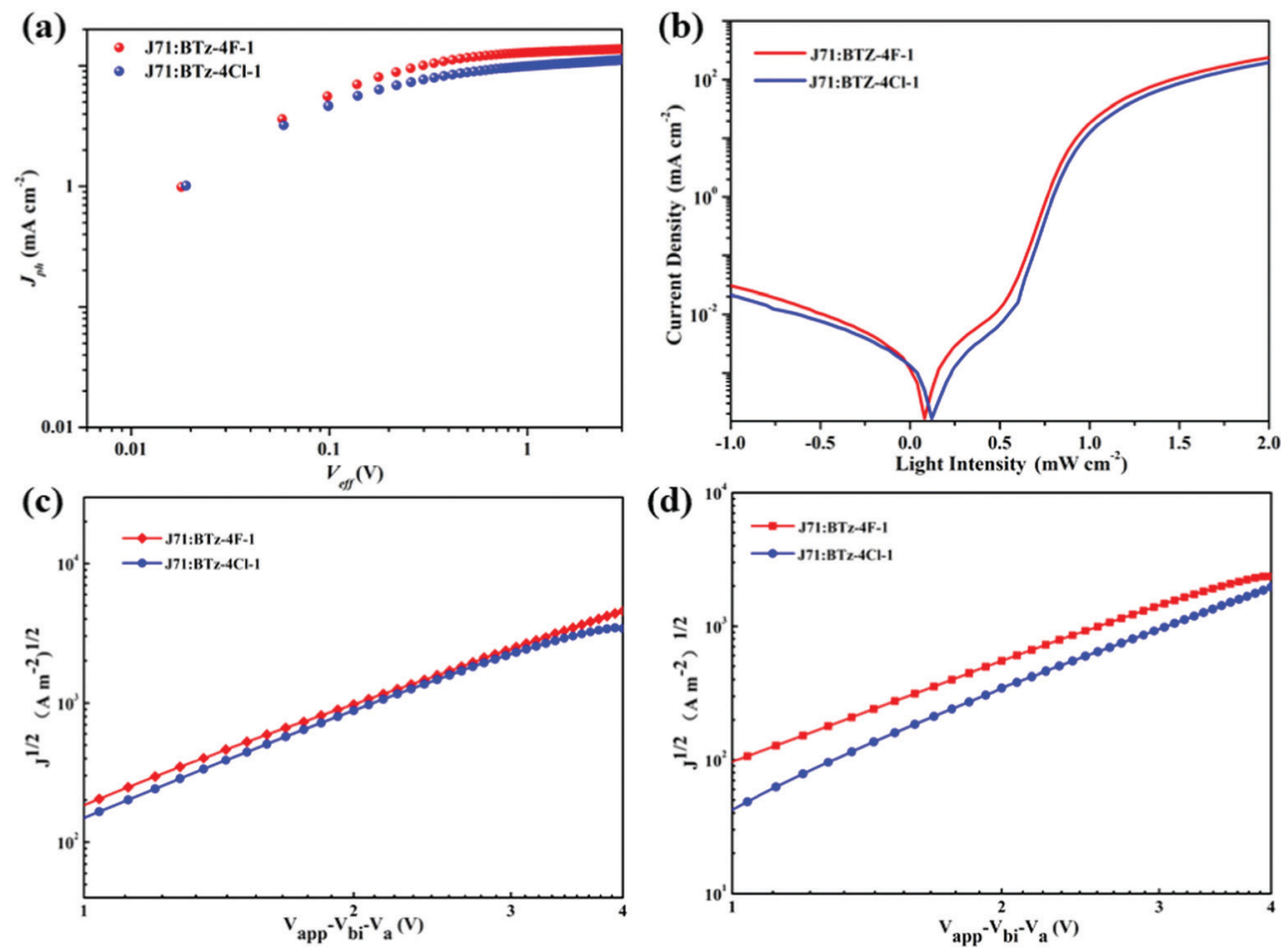

Fig. 6 (a) $J_{\text {ph }}-V_{\text {eff }}$ and (b) dark current density curves of J71: BTz-4F-1 and J71: BTz-4Cl-1 blend OSCs under optimal conditions; $\mathrm{J}^{1 / 2}-V$ plots of the hole-only devices (c) and the electron-only devices (d) based on the J71: BTz-4F-1 and J71: BTz-4Cl-1 blends.

The photocurrent $\left(J_{\mathrm{ph}}\right)$-effective voltage $\left(V_{\text {eff }}\right)$-dark current density $\left(J_{0}\right)$ characteristics were measured for the J71: BTz-4F-1 and J71: BTz-4Cl-1 devices under optimal conditions in order to further evaluate their photoelectric performance, as recorded in



Fig. 7 XRD patterns of the $\mathrm{BTz}-4 \mathrm{~F}-1$ and $\mathrm{BTz}-4 \mathrm{Cl}-1$ pristine solid powders.
Fig. 6. Here, $J_{\mathrm{ph}}$ is defined as $J_{\mathrm{L}}-J_{\mathrm{D}}$, denoting the current under the illumination of $100 \mathrm{~mW} \mathrm{~cm}{ }^{-2}$ and in the dark. $V_{\text {eff }}$ can be described by $V_{\text {eff }}=V_{0}-V_{\text {appl }}$, where $V_{0}$ is the voltage when $J_{\mathrm{ph}}=0$ and $V_{\mathrm{appl}}$ is the applied bias voltage. It is found that $J_{\mathrm{ph}}$ reached saturation when $V_{\text {eff }}$ is up to $3 \mathrm{~V}$. The exciton dissociations ( $\left.\eta_{\text {diss }}\right)$ of the J71: BTz-4Cl-1 and J71: BTz-4F-1 devices are calculated to be $86.44 \%$ and $91.75 \%$, respectively. It implies that J71: BTz-4F-1 devices exhibited more efficient charge dissociation than J71: BTz-4Cl-1 devices, which correlated with the higher $J_{\mathrm{sc}}$ in the J71: BTz-4F-1 devices. On the other hand, a large rectification ratio and low dark current at reverse bias are observed in the optimal J71: BTz-4F-1 devices. It implies that the optimal J71: BTz-4F-1 devices had fewer defects and reduced shunt resistance effect. As a result, the optimal J71: BTz-4Cl-1 devices can get increased $\mathrm{FF}$ and $J_{\mathrm{sc}}$ values.

Fig. 7 shows the powder X-ray diffraction (XRD) patterns of BTz-4F-1 and BTz-4Cl-1. There are two diffraction peaks $(100,010)$ for the BTz-4F-1 powder and three diffraction peaks $(100,200,010)$ for the BTz-4Cl-1 powder. Furthermore, the small-angle diffraction peak of BTz-4Cl-1 is more intense and 


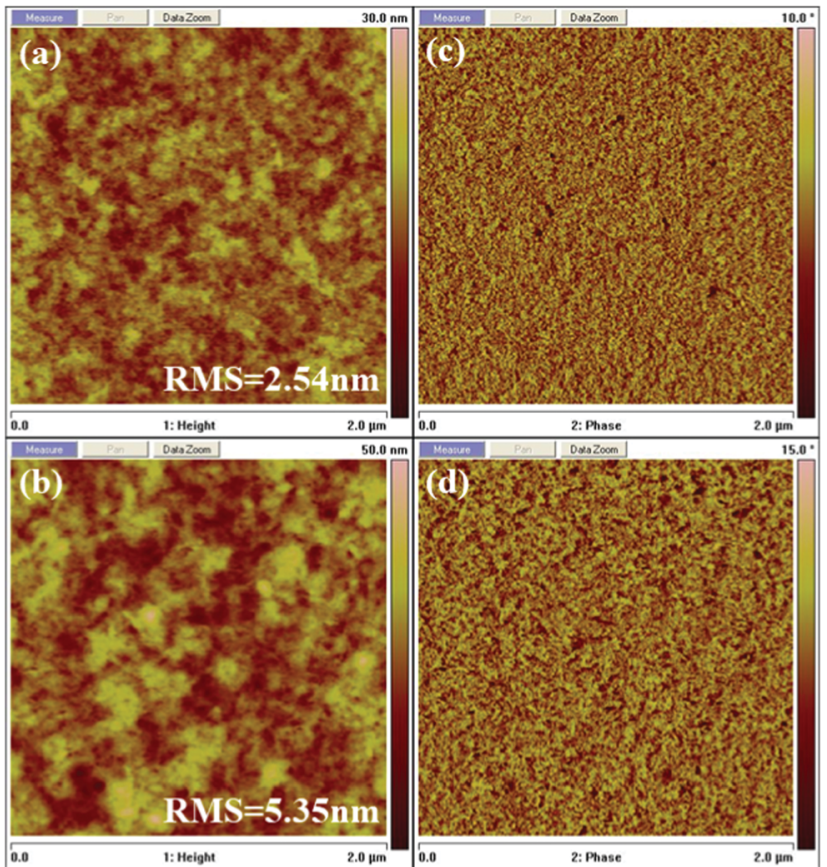

Fig. 8 AFM height $(a, c)$ and AFM phase (b, d) of the optimal J71: BTz-4F-1 $(a, b)$ and $\mathrm{J71}$ : BTz-4Cl-1 (c, d) blend films.

sharper than that of BTz-4F-1, which meant that BTz-4Cl-1 was a more ordered layer stack than $\mathrm{BTz}-4 \mathrm{~F}-1$. Based on their resulting small-angle diffraction peaks respectively at a 2-theta angle $(2 \theta)$ of $5.83^{\circ}$ and $6.08^{\circ}$, the $d$-spacing values are calculated to be $15.13 \AA$ for BTz-4F-1 and $14.51 \AA$ for BTz-4Cl-1 using the Bragg equation, which implies that BTz-4Cl-1 has more ordered lamellar stacking than BTz-4F-1. In addition, based on the weak diffraction peaks in the wide-angle region, the intermolecular $\pi-\pi$ stacking distance is $4.10 \AA$ for $\mathrm{BTz}-4 \mathrm{~F}-1$ and $3.46 \AA$ for BTz-4Cl-1. This further indicates that BTz-4Cl-1 has a stronger intermolecular $\pi-\pi$ stacking effect. Such intermolecular interaction and solubility are observed to further affect the surface morphology of both SMA blends with J71 seriously.

Fig. 8 shows the surface morphologies of J71: BTz-4F-1 and J71: BTz-4Cl-1 blend films under optimal conditions tested using an atomic force microscope (AFM). The root mean square (RMS) roughness of 2.54 and $5.35 \mathrm{~nm}$ were observed in the J71: BTz-4F-1 and J71: BTz-4Cl-1 blend films, respectively. It is obvious that the $\mathrm{J} 71$ : $\mathrm{BTz}-4 \mathrm{~F}-1$ blend film exhibited more appropriate phase separation dimensions than the J71: BTz-4Cl-1 blend film, which is available to promote the dissociation of exciton dissociation and charge transport, resulting in an increasing PCE. ${ }^{45,46}$ It indicates that fluorination instead of chlorination in the terminal here is more available to improve the surface morphologies of the blend films.

\section{Conclusions}

In summary, 4,4'-dimethyl-2,2'-bithiazole and 2,2'-bithiazole were used as $A_{w}$ cores to construct a type of non-fused-ring
SMAs of BTz-4F-0, BTz-4F-1 and BTz-4Cl-1 with an $\mathrm{A}_{\mathrm{W}}(\pi-\mathrm{A})_{2}$ architecture. It was found that $\mathrm{BTz}-4 \mathrm{~F}-1$ and $\mathrm{BTz}-4 \mathrm{Cl}-1$ with methylation in bithiazole core exhibited significantly increasing solubility than the non-methylated BTz-4F-0. Furthermore, the fluorinated BTz-4F-1 exhibited an adjustable crystallinity and increased solubility in comparison with the chlorinated $\mathrm{BTz}-4 \mathrm{Cl}-$ 1. As a result, the J71: BTz-4F-1 based OSCs exhibited better morphological and photovoltaic properties than the J71: BTz4Cl-1 based OSCs. An increasing PCE of 5.72\% was observed in the J71: BTz-4F-1 based OSCs, which is 1.46 times higher than that of the J71: BTz-4Cl-1 based OSCs. This study is of great significance to guide the design and improve the efficiency of simple-structure non-fused-ring SMAs based on an $A_{W}(\pi-A)_{2}$ architecture.

\section{Author contributions}

Jiali Guo: material synthesis, data processing and manuscript preparation. Wei Tang: measurement of OPV properties. Yingshuang Zhang: partial synthesis of materials. Can Qian: partial measurement and analysis. Junfeng Wang: partial data processing. Hua Tan: writing - review \& editing. Weiguo Zhu: supervision for all research work and manuscript revision.

\section{Conflicts of interest}

There are no conflicts of interest to declare.

\section{Acknowledgements}

Thanks to the financial supports from the National Natural Science Foundation of China (51573154, 52073035), the Major Program of the Natural Science Research of Jiangsu Higher Education Institutions (20KJA480004), Jiangsu Provincial Talents Project of High-Level Innovation and Entrepreneurship, the Research Innovation Program for Postgraduate of Jiangsu Province (KYCX18_2617) and the Research Program for Jiangsu Key Laboratories of Environment-Friendly Polymers (PML1901).

\section{References}

1 G. Li, R. Zhu and Y. Yang, Nat. Photonics, 2012, 6, 153-161.

2 C. J. Brabec, S. Gowrisanker, J. J. Halls, D. Laird, S. Jia and S. P. Williams, Adv. Mater., 2010, 22, 3839-3856.

3 Y. Li, Acc. Chem. Res., 2012, 45, 723-733.

4 J. Lee, D. H. Sin, J. A. Clement, C. Kulshreshtha, H. G. Kim, E. Song, J. Shin, H. Hwang and K. Cho, Macromolecules, 2016, 49, 9358-9370.

5 J. Guo, H. Bin, W. Wang, B. Chen, J. Guo, R. Sun, Z.-G. Zhang, X. Jiao, Y. Li and J. Min, J. Mater. Chem. A, 2018, 6, 15675-15683.

6 L. Meng, Y. Zhang, X. Wan, C. Li, X. Zhang, Y. Wang, X. Ke, Z. Xiao, L. Ding and R. Xia, Science, 2018, 361, 1094-1098.

7 Y. Huo, H.-L. Zhang and X. Zhan, ACS Energy Lett., 2019, 4, 1241-1250. 
8 D. Hu, Q. Yang, H. Chen, F. Wobben, V. M. Le Corre, R. Singh, T. Liu, R. Ma, H. Tang, L. J. A. Koster, T. Duan, H. Yan, Z. Kan, Z. Xiao and S. Lu, Energy Environ. Sci., 2020, 13, 2134-2141.

9 X. Ma, A. Zeng, J. Gao, Z. Hu, C. Xu, J. H. Son, S. Y. Jeong, C. Zhang, M. Li, K. Wang, H. Yan, Z. Ma, Y. Wang, H. Y. Woo and F. Zhang, Natl. Sci. Rev., 2021, 8, nwaa305.

10 Y. Wang, F. Wang, J. Gao, Y. Yan, X. Wang, X. Wang, C. Xu, X. Ma, J. Zhang and F. Zhang, J. Mater. Chem. C, 2021, 9, 9892-9898.

11 B. Kan, H. Feng, X. Wan, F. Liu, X. Ke, Y. Wang, Y. Wang, H. Zhang, C. Li, J. Hou and Y. Chen, J. Am. Chem. Soc., 2017, 139, 4929-4934.

12 F. Liu, Z. Zhou, C. Zhang, T. Vergote, H. Fan, F. Liu and X. Zhu, J. Am. Chem. Soc., 2016, 138, 15523-15526.

13 Y. Lin, Q. He, F. Zhao, L. Huo, J. Mai, X. Lu, C. J. Su, T. Li, J. Wang, J. Zhu, Y. Sun, C. Wang and X. Zhan, J. Am. Chem. Soc., 2016, 138, 2973-2976.

14 M. Li, Y. Liu, W. Ni, F. Liu, H. Feng, Y. Zhang, T. Liu, H. Zhang, X. Wan, B. Kan, Q. Zhang, T. P. Russell and Y. Chen, J. Mater. Chem. A, 2016, 4, 10409-10413.

15 Y. Lin, J. Wang, Z. G. Zhang, H. Bai, Y. Li, D. Zhu and X. Zhan, Adv. Mater., 2015, 27, 1170-1174.

16 H. You, S. Lee, D. Kim, H. Kang, C. Lim, F. S. Kim and B. J. Kim, ACS Appl. Mater. Interfaces, 2021, 13, 47817-47825.

17 X. Li, C. Li, L. Ye, K. Weng, H. Fu, H. S. Ryu, D. Wei, X. Sun, H. Y. Woo and Y. Sun, J. Mater. Chem. A, 2019, 7, 19348-19354.

18 H. Huang, Q. Guo, S. Feng, C. Zhang, Z. Bi, W. Xue, J. Yang, J. Song, C. Li, X. Xu, Z. Tang, W. Ma and Z. Bo, Nat. Commun., 2019, 10, 3038.

19 Q. Zhu, D. Liu, Z. Lu, C. Gu, K. Zhang, X. Bao, Q. Li and R. Yang, J. Mater. Chem. A, 2019, 7, 4823-4828.

20 D. Li, X. Zhang, D. Liu and T. Wang, J. Mater. Chem. A, 2020, 8, 15607-15619.

21 G. Chai, Y. Chang, J. Zhang, X. Xu, L. Yu, X. Zou, X. Li, Y. Chen, S. Luo, B. Liu, F. Bai, Z. Luo, H. Yu, J. Liang, T. Liu, K. S. Wong, H. Zhou, Q. Peng and H. Yan, Energy Environ. Sci., 2021, 14, 3469-3479.

22 W. Peng, G. Zhang, L. Shao, C. Ma, B. Zhang, W. Chi, Q. Peng and W. Zhu, J. Mater. Chem. A, 2018, 6, 24267-24276.

23 C. e. Zhang, S. Ming, H. Wu, X. Wang, H. Huang, W. Xue, X. Xu, Z. Tang, W. Ma and Z. Bo, J. Mater. Chem. A, 2020, 8, 22907-22917.

24 T. Duan, M. Babics, A. Seitkhan, Y. Firdaus, R.-Z. Liang, F. Cruciani, S. Liu, S. Lopatin and P. M. Beaujuge, J. Mater. Chem. A, 2018, 6, 9368-9372.

25 J. Pan, Y. Shi, J. Yu, H. Zhang, Y. Liu, J. Zhang, F. Gao, X. Yu, K. Lu and Z. Wei, ACS Appl. Mater. Interfaces, 2021, 13, 22531-22539.

26 J. Ahn, S. Oh, H. Lee, S. Lee, C. E. Song, H. K. Lee, S. K. Lee, W. W. So, S. J. Moon, E. Lim, W. S. Shin and J. C. Lee, ACS Appl. Mater. Interfaces, 2019, 11, 30098-30107.
27 W. Peng, G. Zhang, M. Zhu, H. Xia, Y. Zhang, H. Tan, Y. Liu, W. Chi, Q. Peng and W. Zhu, ACS Appl. Mater. Interfaces, 2019, 11, 48128-48133.

28 M. Chang, L. Meng, Y. Wang, X. Ke, Y.-Q.-Q. Yi, N. Zheng, W. Zheng, Z. Xie, M. Zhang, Y. Yi, H. Zhang, X. Wan, C. Li and Y. Chen, Chem. Mater., 2020, 32, 2593-2604.

29 Z. X. Liu, Z. P. Yu, Z. Shen, C. He, T. K. Lau, Z. Chen, H. Zhu, X. Lu, Z. Xie, H. Chen and C. Z. Li, Nat. Commun., 2021, 12, 3049.

30 Y. Zhang, X. Zheng, W. Tang, W. Peng, H. Xia, J. Zhu, M. Zhu, W. Chi, H. Tan and W. Zhu, Dyes Pigm., 2020, 177, 108319.

31 Y. N. Chen, M. Li, Y. Wang, J. Wang, M. Zhang, Y. Zhou, J. Yang, Y. Liu, F. Liu, Z. Tang, Q. Bao and Z. Bo, Angew. Chem., Int. Ed., 2020, 59, 22714-22720.

32 T. J. Wen, Z. X. Liu, Z. Chen, J. Zhou, Z. Shen, Y. Xiao, X. Lu, Z. Xie, H. Zhu, C. Z. Li and H. Chen, Angew. Chem., Int. Ed., 2021, 60, 12964-12970.

33 J. Zhu, X. Zheng, H. Tan, H. Tan, J. Yang, J. Yu and W. Zhu, Chem. Phys. Lett., 2019, 726, 7-12.

34 Q. Liu, K. Jin, W. Li, Z. Xiao, M. Cheng, Y. Yuan, S. Shi, Z. Jin, F. Hao, S. Yang and L. Ding, J. Mater. Chem. A, 2020, 8, 8857-8861.

35 J. Wang, J. Zhang, Y. Xiao, T. Xiao, R. Zhu, C. Yan, Y. Fu, G. Lu, X. Lu, S. R. Marder and X. Zhan, J. Am. Chem. Soc., 2018, 140, 9140-9147.

36 S. Yu, Y. Chen, L. Yang, P. Ye, J. Wu, J. Yu, S. Zhang, Y. Gao and H. Huang, J. Mater. Chem. A, 2017, 5, 21674-21678.

37 Y. Firdaus, Q. He, Y. Lin, F. A. A. Nugroho, V. M. Le Corre, E. Yengel, A. H. Balawi, A. Seitkhan, F. Laquai, C. Langhammer, F. Liu, M. Heeney and T. D. Anthopoulos, J. Mater. Chem. A, 2020, 8, 1164-1175.

38 B. Qiu, L. Xue, Y. Yang, H. Bin, Y. Zhang, C. Zhang, M. Xiao, K. Park, W. Morrison, Z.-G. Zhang and Y. Li, Chem. Mater., 2017, 29, 7543-7553.

39 M. Li, Z. Li, Z. Yang, Z. Liu, K. Zhang, L. Yang, Q. Peng, W. Zhu and Y. Liu, Dyes Pigm., 2019, 170, 107595.

40 J. Li, Y. Wang, Z. Liang, J. Qin, M. Ren, J. Tong, C. Yang, C. Yang, X. Bao and Y. Xia, J. Mater. Chem. C, 2020, 8, 2483-2490.

41 H. Tan, H. Tan, X. Zheng, J. Yang, J. Yu and W. Zhu, J. Mater. Chem. C, 2020, 8, 3183-3191.

42 G. P. Kini, S. J. Jeon and D. K. Moon, Adv. Mater., 2020, 32, e1906175.

43 C. Xu, K. Jin, Z. Xiao, Z. Zhao, X. Ma, X. Wang, J. Li, W. Xu, S. Zhang, L. Ding and F. Zhang, Adv. Funct. Mater., 2021, 31, 2107934.

44 H. Tan, X. Zheng, J. Zhu, J. Yu and W. Zhu, J. Mater. Chem. C, 2019, 7, 13301-13306.

45 Y. Zhou, K. L. Gu, X. Gu, T. Kurosawa, H. Yan, Y. Guo, G. I. Koleilat, D. Zhao, M. F. Toney and Z. Bao, Chem. Mater., 2016, 28, 5037-5042.

46 X. Ke, L. Meng, X. Wan, Y. Cai, H.-H. Gao, Y.-Q.-Q. Yi, Z. Guo, H. Zhang, C. Li and Y. Chen, Nano Energy, 2020, 75, 104988. 